\title{
FACTORES QUE DETERMINAN LA MOROSIDAD DE TARJETAS DE CRÉDITO DE UNA EMPRESA ESPECIALIZADA EN CRÉDITOS DE CONSUMO - 2011
}

\section{FACTORS THAT DETERMINE THE DELINQUENCY OF CREDIT CARDS OF A COMPANY SPECIALIZED IN CONSUMER CREDITS -2011}

\section{MG. WALTER ADRIÁN LANDEO TORRES}

\section{RESUMEN}

Objetivo: Estimar un modelo que permita evaluar de la mejor forma posible a aquellos que solicitan una tarjeta de crédito, estableciendo los principales factores que afectan la probabilidad de que un cliente sea moroso o no. Método: 1) Para realizar la investigación, se ha empleado información secundaria y primaria. 2) Se utilizó el método estadístico Credit Scoring, aplicando sus opciones de estimación Probit y Logit. Considerando la limitante de contar con solo un periodo de observación se aplicó la metodología de corte transversal. 3) Se especificaron variables independientes que recogen tanto factores cuantitativos como cualitativos, que influyen y o determinan la morosidad. 4) Se utilizó paquetes econométricos y/o estadístico, y ha permitido determinar las principales características de cada serie como media, varianza, valores mínimos y máximos; Comparación de correlaciones entre variables. Resultados: 1) Se observa que las personas de mayores ingresos consumen más, sin embargo, tienen mayor cuidado en el pago de sus cuotas. 2) Contrario a la intuición, resulta que el mayor plazo del préstamo afectaría negativamente el comportamiento de los prestatarios. 3) De la serie Antigüedad Laboral se observa que estas personas al estar inmersos en el mercado laboral por más tiempo tendrían mayor estabilidad en sus recursos y resultando ser mejores pagadores.4) La variable Edad del deudor afecta negativamente la morosidad o la probabilidad de mora disminuye a medida que el deudor avanza en edad. 5) La serie Estado Civil sugiere que los solteros son más propensos a ser malos pagadores. 6) De la variable categoría laboral se puede inferir que los dependientes, suelen ser mejores pagadores. 9) También se nota que la tarjeta habiente que tienen más de una tarjeta de crédito resultan ser mejores clientes que aquellos que poseen menos tarjetas de crédito. Conclusiones: Los factores relevantes para determinar la probabilidad de morosidad en las tarjetas de crédito: la edad, el nivel socioeconómico, categoría laboral, número de cuotas, tasa de interés, antigüedad laboral y la relación saldo de deuda sobre ingresos pero fundamentalmente la variable clase.

Palabras claves: Tarjeta de crédito, Morosidad, crédito de consumo

\section{ABSTRACT}


Objective: To estimate a model to evaluate the best possible way those who apply for a credit card, establishing the main factors affecting the likelihood that a customer is late or not.

Method: 1) To conduct the research, it has been used primary and secondary information. 2) the statistical method was used credit scoring, applying their options probit and logit estimation. Considering the limitation of having only an observation period cross-sectional methodology was applied. 3) independent variables that collect both quantitative and qualitative factors, which influence or determine and delinquencies were specified. 4) econometric packages and / or statistical was used, and has identified the main features of each series as mean, variance, minimum and maximum values; Comparison of correlations between variables. Results: 1) It is observed that people with higher incomes consume more, but are more careful in paying their dues. 2) Contrary to intuition, it is that the biggest loan term negatively affect the behavior of borrowers. 3) From Antiquity Laboral series shows that these people are immersed in the labor market for longer would have more stability in their resources and turning out to be better payers 4) Variable Age of debtor default or negatively affects the probability of default decreases as the debtor gets older. 5) The State Civil series suggests that singles are more likely to be bad payers. 6) employment status variable can be inferred that the dependents are generally better payers. 9) Also note that cardholders who have more than one credit card customers turn out to be better than those who have fewer credit cards. Conclusions: Relevant to determine the probability of default on credit cards factors: age, socioeconomic status, employment status, number of payments, interest rate, seniority and the balance ratio of debt to income but primarily the variable class.

Autor: Mg. Walter Adrián Landeo Torres

Docente de la Universidad Alas Peruanas Facultad de Ciencias Empresariales y Educación

\section{INTRODUCCIÓN}

Uno de los instrumentos crediticios que de crédito, que muchas veces el en forma más dinámica en la moderna sociedad de consumo es la tarjeta de crédito. Su difusión ha permitido a los consumidores adquirir en forma ágil y ventajosa diversos bienes y servicios. $\mathrm{Al}$ mismo tiempo, como sociedad se ha creado tanta dependencia en las tarjetas consumidor no se percata que si paga a través de este medio, el dinero que gane en el futuro será destinado a pagar las cosas que adquirió en el pasado.

En el Perú, las tarjetas de crédito muestran una evolución creciente, y son emitidas por instituciones financieras que 
en algunos casos se encuentran afiliados a operadores internacionales, como: Visa, Mastercard, y American express. Así encontramos tanto tarjetas de marca propia, como de marca privada o compartida, como: tarjeta válida plaza vea, CMR Falabella/Financiera CMR, Ripley/financiera cordillera y Banco del trabajo que a través de Netcard trabajó con 60 empresas (Metro, Hiraoka, Correlo, Alda, etc.)

El incremento alcanzado, se explica principalmente por la expansión de su mercado objetivo a los niveles socioeconómicos (NSE) C y D. La explosión en la emisión de tarjetas de crédito que empezó a mediados de la década de los noventa generó el incremento de la morosidad. Este incremento se explica principalmente por el desconocimiento del comportamiento y características del cliente y el bajo nivel de cultura crediticia de éstos.

El uso de las tarjetas de crédito se ha incrementado considerablemente en nuestro país y en muchos casos las personas no ponen reparos al endeudarse. Sin duda esta "moneda plástica" se ha convertido en un medio eficaz de pago y de la obtención de crédito de forma inmediata.

Sin embargo, actualmente, el índice de morosidad de los clientes de tarjetas de crédito no es tan alto como el de años anteriores, debido a que las instituciones financieras cuentan con mayor información acerca del mercado objetivo, lo que ha permitido mejorar los sistemas de riesgo. Además, hoy en día, los usuarios cuentan con mayor información pública.

Con esta investigación se pretende estimar un modelo que permita evaluar mejor a los que solicitan una tarjeta de crédito, estableciendo los principales factores que determinan la morosidad y los resultados que se esperan permita hacer más objetiva la evaluación de tarjetas de crédito y contribuyan a la elaboración de buenas políticas crediticias.

Considerando la limitante de contar con solo un periodo de observación se aplicó la metodología de corte transversal (máxima verosimilitud).

$\mathrm{Se} \quad$ especificaron variables independientes que recogen tanto 
factores cuantitativos como cualitativos, que influyen y o determinan la morosidad. La variable dependiente, es una variable dicotómica, que se denominará "morosidad", y que para explicarla se considera el siguiente criterio:

$0=$ Cumple con sus pagos (días de atraso hasta 30 días)

1 = Mal pagador (días de atraso mayor a 30 días)

Se utilizó paquetes econométricos y/o estadístico, cuyo procedimiento aplicado ha permitido: determinar las principales características de cada serie como media, varianza, valores mínimos y máximos; Comparación de correlaciones entre variables; construcción de modelos logit y probit con las variables más relevantes; evaluación de los modelos.

Utilizando los paquetes econométricos, debido a su potencia en la carga de una base de datos grande, el procedimiento general aplicado es similar al planteado por Matsuoka y Barrientos, ya que para este tipo de análisis es común:

1) Análisis univariado con el fin de determinar las principales características de cada serie como media, varianza, valores mínimos y máximos.

2) Comparación de correlaciones entre variables mediante la matriz de correlaciones, a fin de determinar la relación existente entre cada una de las variables explicativas con la endógena $\mathrm{y}$ de eliminar posibles problemas de multicolinealidad en la estimación del modelo, si dos variables están muy correlacionadas $(>=0.9)$ se evitará incluir una de ellas.

3) Construcción de modelos logit y probit con las variables más relevantes. Con la finalidad de obtener un modelo significativo se procede a descartar variables, conforme a la que presente en cada regresión la probabilidad de estadístico $\mathrm{Z}$ más alta. De esta manera, se realiza la incorporación y descarte de series hasta llegar a un modelo en el que todas las variables explicativas son significativas.

4) Evaluación de los modelos obtenidos, para lo cual:

- Se verificó que el signo de los coeficientes de cada serie tenga sentido con la probabilidad de ser mal pagador.

- Se evaluó la significancia estadística individual de los paramétricos de acuerdo al estadístico $\mathrm{Z} \quad \mathrm{y} \quad \mathrm{su}$ probabilidad correspondiente.

- Se generó la tabla de predicción esperada para determinar el poder del modelo.

5) Selección del modelo óptimo. 


\section{RESULTADOS}

3.4.1) Análisis univariado (estadística descriptiva)

De la información que se desprende de las tablas 1 y 2 del anexo $\mathrm{D}$, se puede establecer las primeras diferencias entre los préstamos vigentes y vencidos.

Así, tal como resulta lógico suponer la tasa de interés promedio es mayor en el caso de los préstamos vencidos, denotándose que a mayor tasa de interés la probabilidad de incumplimiento aumenta.

Se observa que el promedio del monto o saldo del préstamo resulta ser mayor cuando se trata de préstamos vigentes al asociar esta variable con el nivel de ingresos de los prestatarios, tenemos que las personas de mayores ingresos consumen más, sin embargo, tienen mayor cuidado en el pago de sus cuotas (por lo que se encuentran al día en sus compromisos), por lo tanto, se encuentra dentro de la cartera de colocaciones vigente.

Relacionado el párrafo precedente, de las referidas tablas también se observa que en la cartera vigente se encuentra concentrada en los niveles socioeconómicos más altos (A y B), y no en los estratos económicos de menores recursos.
Así también se puede anotar que la variable Clase de un deudor es mayor (1 es mejor, 9 es peor) cuando registra su deuda como vencida que cuando se encuentra vigente.

De otro lado, contrario a la intuición, resulta que el número de cuotas promedio de los créditos en situación vigente es menor cuando nos referimos a la cartera morosa, es decir el mayor plazo del préstamo afectaría negativamente el comportamiento de los prestatarios.

En cuanto a la variable Ingreso, como resulta lógico pensar, el monto promedio de los ingresos es mayor cuando se trata de préstamos en situación de vigentes, lo que sugiere su influencia en el momento del pago puntual de las cuotas.

De la serie Antigüedad Laboral se observa que es mayor, en promedio, cuando se trata de prestatarios incluidos en la cartera de colocaciones vigentes lo que denota que estas personas al estar inmersos en el mercado laboral por mas tiempo tendrían mayor estabilidad en sus recursos (mas confiables, madurez con ahorros) resultando ser mejores pagadores que aquellos que recién empiezan a laborar.

La variable Edad en promedio es mayor cuando se trata de la cartera de 
colocaciones vigente lo cual sugiere que a mayor edad las personas tienden a ser más responsables en el cumplimiento de sus deudas. Es decir, que la edad del deudor afecta negativamente la morosidad o la probabilidad de mora disminuye a medida que el deudor avanza en edad. Al analizar la edad de los deudores con sus probabilidades de mora se encuentra que aproximadamente hasta los 27 años la contribución marginal de la edad para explicar el evento es positiva, en tanto que, a partir de la edad señalada, la explicación del evento mediante la edad se vuelve negativa.

Al observar la variable Lima, se nota que arroja resultados similares cuando se compara ambas carteras de créditos, esto se puede explicar ya que esta variable constituye el 98\% (ver tabla 1 del anexo E) de la población total por lo que es bastante significativa para las dos carteras bajo comentario.

La serie Estado Civil que distingue sólo dos condiciones (solteros y no solteros) sugiere que los solteros son más propensos a ser malos pagadores que aquellos que son casados, viudos o separados. Otra variable que agrupa dos situaciones es la categoría laboral (distingue entre dependientes y no dependientes) de la cual se puede inferir que las tarjetas habientes cuya situación laboral es la de dependiente, suelen ser mejores pagadores que aquellos que se encuentran dentro de otro régimen laboral; sin embargo, es de anotar que la condición dependiente representa el 85\% del total de la población (ver tabla 2 del anexo E) por lo que se puede advertir un sesgo relevante.

De este análisis, aparentemente los hombres resultan ser mejores pagadores que las mujeres, esta relación es relativamente importante ya que los hombres representan el $47 \%$ del universo poblacional (ver tabla 3 del anexo E). La variable Tarjeta por Tarjeta es mayor en promedio cuando se observa la cartera vigente lo cual puede sugerir que los prestatarios analizados habrían obtenido esta tarjeta de crédito considerando la evaluación previa facilitada en el análisis de otra institución financiera, es decir, resultarían ser mejores pagadores aquellos clientes que han tenido una tarjeta de crédito.

También se nota que la tarjeta habiente que tienen más de una tarjeta de crédito resultan ser mejores clientes que aquellos que poseen menos tarjetas de crédito, esto puede estar ligado al nivel de ingresos de los prestatarios, es decir tendrán mas tarjetas de crédito aquellos que presenten mayores ingresos existiendo una relación negativa en 
cuanto a la morosidad con el número de tarjetas de crédito.

Tabla 1: Estadística descriptiva de la Cartera de Créditos Morosa

\begin{tabular}{|l|c|c|c|c|c|}
\hline \multicolumn{1}{|c|}{} & $\mathrm{N}$ & Minimum & Maximum & Mean & Std. Desv \\
\hline SEX & 12482 & 0 & 1 & 0.418 & 0.49 \\
LIMA & 12482 & 0 & 1 & 0.99 & 0.09 \\
CAT_LAB & 12482 & 0 & 1 & 0.11 & 0.32 \\
EDAD & 12482 & 18 & 75 & 35.77 & 11.17 \\
TASA & 12482 & 62.27 & 90.12 & 69.13 & 7.06 \\
NSE_N & 12482 & 0 & 2 & 0.69 & 0.77 \\
NSE_AB & 12482 & 0 & 1 & 0.19 & 0.40 \\
NSE_C & 12482 & 0 & 1 & 0.31 & 0.46 \\
NSE_DE & 12482 & 0 & 1 & 0.49 & 0.50 \\
EST_CIV & 12482 & 0 & 1 & 0.39 & 0.49 \\
ANT_LAB & 12482 & 0 & 40 & 5.75 & 6.03 \\
NUM_CUO & 12482 & 1 & 39 & 9.88 & 9.51 \\
SALDO & 12482 & 0.1 & 25724.1 & 739.95 & 1118.37 \\
TXT & 12482 & 0 & 1 & 0.23 & 0.42 \\
INGRESO & 12482 & 300 & 39000 & 1451.92 & 2032.41 \\
NUM_TARJ & 12482 & 0 & 6 & 0.41 & 0.65 \\
SAL_ING & 12482 & 0.00094429 & 2077.9373 & 65.30 & 73.46 \\
CLASE & 12482 & 2 & 9 & 5.80 & 1.13 \\
\hline
\end{tabular}

Tabla 2: Estadística descriptiva de la Cartera de Créditos Vigente

\begin{tabular}{|l|c|c|c|c|c|}
\hline \multicolumn{1}{l|}{} & $\mathrm{N}$ & Minimum & Maximum & Mean & Std. Desv \\
\hline SEX & 318182 & 0 & 1 & 0.47 & 0.50 \\
LIMA & 318182 & 0 & 1 & 0.99 & 0.11 \\
CAT_LAB & 318182 & 0 & 1 & 0.15 & 0.36 \\
EDAD & 318182 & 18 & 75 & 39.04 & 12.04 \\
TASA & 318182 & 62.27 & 90.7 & 67.93 & 6.52 \\
NSE_N & 318182 & 0 & 2 & 0.87 & 0.81 \\
NSE_AB & 318182 & 0 & 1 & 0.28 & 0.45 \\
NSE_C & 318182 & 0 & 1 & 0.32 & 0.47 \\
NSE_DE & 318182 & 0 & 1 & 0.40 & 0.49 \\
EST_CIV & 318182 & 0 & 1 & 0.46 & 0.50 \\
ANT_LAB & 318182 & 0 & 40 & 6.82 & 7.19 \\
NUM_CUO & 318182 & 1 & 40 & 9.20 & 10.06 \\
SALDO & 318182 & 0 & 77304 & 875.76 & 465.07 \\
TXT & 318182 & 0 & 1 & 0.31 & 0.46 \\
INGRESO & 318182 & 107 & 49800 & 1662.68 & 2064.54 \\
NUM_TARJ & 318182 & 0 & 8 & 0.53 & 0.76 \\
SAL_ING & 318182 & 0 & 14583.33 & 74.57 & 103.57 \\
CLASE & 318182 & 1 & 9 & 2.45 & 1.12 \\
\hline
\end{tabular}

ANEXO E: Estadísticas 
Tabla 1: Estadística de la Morosidad por lugar de residencia (Lima o fuera de Lima)

\begin{tabular}{|ccc|}
\hline MOROSO & LIMA & PORCENTAJE \\
\hline \multirow{3}{*}{ 0: No Moroso } & 0: Fuera de Lima & 1.1 \\
& 1: Dentro de Lima & 95.1 \\
& total & 96.2 \\
\hline \multirow{3}{*}{ 1: Moroso } & 0: Fuera de Lima & 0.0 \\
& 1: Dentro de Lima & 3.7 \\
& total & 3.8 \\
\hline \multirow{2}{*}{ Total } & 0: Fuera de Lima & 1.2 \\
& 1: Dentro de Lima & 98.8 \\
& total & 100.0 \\
\hline
\end{tabular}

Tabla 2: Estadística de la Morosidad por Categoría Laboral

\begin{tabular}{|clc|}
\hline MOROSO & CAT_LAB & PORCENTAJE \\
\hline \multirow{3}{*}{ 0: No Moroso } & 0: Dependiente & 81.9 \\
& 1: No dependiente & 14.4 \\
& total & 96.2 \\
\hline \multirow{3}{*}{ 1: Moroso } & 0: Dependiente & 3.3 \\
& 1: No dependiente & 0.4 \\
& total & 3.8 \\
\hline \multirow{2}{*}{ Total } & 0: Dependiente & 85.2 \\
& 1: No dependiente & 14.8 \\
& total & 100.0 \\
\hline
\end{tabular}

Tabla 3: Estadística de la Morosidad por Sexo

\begin{tabular}{|ccc|}
\hline MOROSO & SEX & PORCENTAJE \\
\hline \multirow{3}{*}{ 0: No Moroso } & 0: Mujeres & 50.5 \\
& 1: Hombres & 45.7 \\
& total & 96.2 \\
\hline \multirow{3}{*}{ 1: Moroso } & 0: Mujeres & 2.2 \\
& 1: Hombres & 1.5 \\
& total & 3.8 \\
\hline \multirow{2}{*}{ Total } & 0: Mujeres & 52.8 \\
& 1: Hombres & 47.2 \\
& total & 100.0 \\
\hline
\end{tabular}

\section{DISCUSIÓN}

El método empleado pretende recoger la mejor estimación del modelo de regresión. Como consecuencia, un modelo bien diseñado por ejemplo, debe dar un elevado porcentaje de puntajes altos (riesgo bajo), para aquellos créditos que se comportarán bien (serán devueltos en tiempo y forma). Sin embargo, como sabemos que ningún modelo es perfecto, algunos préstamos malos recibirán un puntaje más alto que algunos préstamos buenos. Ningún modelo nos dirá con certeza absoluta cuál será el comportamiento crediticio 
futuro de un individuo en particular, pero un modelo como el planteado debe ser capaz de predecir con exactitud el comportamiento promedio de créditos otorgados a grupos de individuos que comparten condiciones o características similares que han sido identificadas por su importancia, como relevantes para la calidad crediticia.

El estudio ha permitido hacer evidente que la importancia de las siguientes variables: nivel socioeconómico, categoría laboral, número de cuotas, edad, tasa de interés, clase interna, antigüedad laboral, y la relación saldo de deuda sobre los ingresos mensuales, donde la categoría laboral, plazo del préstamo, la tasa de interés pactada, la clase y la participación del saldo del crédito sobre el nivel de ingresos afecta positivamente $y$ el nivel socioeconómico, la edad y el número de años laborales inciden negativamente en la probabilidad de incumplimiento de los

\section{CONCLUSIONES}

1. Los resultados de las estimaciones estadísticas y econométricas realizadas permiten concluir, que los factores relevantes para determinar la probabilidad de morosidad en las tarjetas de crédito en esta institución financiera son: la edad, el nivel socioeconómico, categoría laboral, número de cuotas, tasa pagos. Bajo tal escenario, las políticas crediticias que se implementen con la finalidad de disminuir los índices de morosidad de la cartera de tarjetas de crédito deberán considerar estas variables.

Un análisis similar al seguido en este trabajo, se realizó en un estudio de Matsuoka y Barrientos, en el que la variable de ingresos resulta ser la más significativa, así también obtienen que las variables como tenencia de teléfono, el estado civil y el lugar de residencia explican la morosidad de los préstamos personales del BWS. Para efectos del trabajo de investigación, esta variable no resulta significativa, sin embargo se puede decir que forma parte de otras dos variables que son importantes dentro del modelo, como por ejemplo nivel socioeconómico (basados en la definición de la variable) y la relación del saldo de la deuda sobre el nivel de ingresos.

de interés, antigüedad laboral y la relación saldo de deuda sobre ingresos pero fundamentalmente la variable clase. De esta manera, se puede decir que implementación de un sistema de scoring, como una herramienta adicional (ya que actualmente realiza la evaluación de las solicitudes de crédito en forma 
manual), debido a que permitiría conocer exante la probabilidad de default en los pagos de las cuotas de las tarjetas de crédito concedida. Además a medida que se mejore el sistema de información de la institución (que va desde el buen llenado de las solicitudes de créditos hasta el seguimiento del comportamiento crediticio de los deudores, y que implica nuevas o variables más desagregadas), se podrá ir afinando el modelo planteado para que los resultados sean más robustos.

2. A pesar del incremento del mercado de tarjetas de crédito, que en el último año presenta una variación de s/ 591 millones, en el Perú este mercado aún es pequeño y concentrado, por lo que existe un gran potencial de crecimiento en los estratos de la población más desatendidos (NSE C y D).

3. Para incrementar esta cartera sin aumentar los niveles de morosidad es necesario que las entidades amplíen su conocimiento sobre el comportamiento de los clientes e implementen estrategias de segmentación, que respondan a las necesidades de los clientes. En este sentido, se recomienda:

- Desarrollar un mayor conocimiento del perfil del mercado objetivo, sobre todo de los segmentos NSE C y D, para establecer cómo obtienen sus ingresos, su comportamiento crediticio y sus necesidades.

- Realizar análisis estadísticos con la información histórica que poseen, de tal manera que se identifiquen parámetros de riesgo, estableciendo quiénes son morosos en función de la edad, sexo o el nivel de ingresos, etc.

- Mediante la aplicación de modelos econométricos, determinar la probabilidad de pago del cliente o el nivel de correlación éxito-fracaso en el pago.

- Mejorar los sistemas de información y de administración de créditos, con políticas de evaluación, y de cobranza (tanto morosa como preventiva) claramente definidas.

- Expandir la cultura crediticia, las entidades deberán educar financieramente a sus clientes, e incrementar el nivel de bancarización.

4. Se debe hacer notar que los resultados obtenidos no resultan ser aplicables o extensivos a otras entidades del sistema financiero peruano porque sólo se ha analizado la información de una sola entidad especializada en créditos de consumo, y dado que sólo contamos con información de un periodo de tiempo, este modelo tendrá una vigencia relativa mientras las condiciones sobre las que se tomaron los datos no cambien. Es decir, 
resultan necesarias las actualizaciones y verificaciones de información para que

\section{REFERENCIAS BIBLIGRÁFICAS}

1. ALLAIN, Luis (2000). Banca Electrónica: Posibilidades, Riesgos y Lineamientos Regulatorios. SBS, Lima.

2. BANCO CENTRAL DE RESERVA DEL PERÚ (2002). El costo del Crédito en el Perú.

3. BRITO, Dagobort \& HARTLEY, Peter (1995). Consumer Rationality and credit cards.

4. CANNER, Glenn \& LUCKETT, Charles (1992). Development in the pricing of credit card services. FED.RES.BULL.

5. CARBONEL, P. (1999). La Protección del Consumidor Titular de Tarjetas de Pago en la Comunidad Europea. Madrid.

6. CARGILL, Thomas \& WENDELL, Jeanne (1996). Bank credit cards: consumer irrationality versus market fortes.

7. GETE-ALONSO, MARÍA DEL CARMEN (1990). El Pago mediante Tarjetas de Crédito. La Ley. Madrid.

8. MATSUOKO, Aldo y BARRIENTOS, Fernando (2002). Metodología para el se vaya ajustando el modelo y responda a los factores actuales.

monitoreo de la cartera de banca personal sobre la base del credit scoring: caso banco Wiese Sudameris. Uinversidad del Pacífico.

9. MESTER, Loreta (1997). Qué es el crédito scoring ?

10. OZDEMIR, Ozlemy y LEVENT, Boran (2001). Examing credit default risks: An empirical study of consumer credit clients. Yeditepe University. Estambul.

11. PARAIZAMÁN, ROBERTO (2003). El Crédito de Consumo en el Perú. CIUP. Lima.

12. SCHREINER, Mark (2001). Arboles estadísticos en las microfinanzas. BCRD.

13. WENDEL, JEANNE (1996). Bank Credit Cards: Consumer Irrationality versus Market Forces. J. CONSUMER AFF.

14. ZAPATA, LUIS (2002). "Masificación en marcha". Semana económica. Vol. 808.

15. ZYWICKI, TODD (1999). Economics of Credit Cards. George Mason University School of Law. 
